\title{
Transmissions de l'exploitation en moyenne montagne pyrénéenne : ruptures et continuités
}

\author{
Claire Manoli', Jean-Philippe Choisis², \\ Sylvie Ladet ${ }^{3}$, Annick Gibon ${ }^{4}$
}

\section{Introduction}

Que transmet-on quand on transmet une exploitation agricole aujourd'hui ? La pérennité des exploitations et la transmission intergénérationnelle sont au centre des enjeux du développement durable, en agriculture notamment (Landais, 1999). L'évolution de l'agriculture française, mais aussi européenne, est marquée par une diminution du nombre d'exploitations (la population agricole a en effet été divisée par trois depuis les années 1980 [Gambino et al., 2012]), ainsi que le vieillissement de la population active. Le secteur agricole est ainsi le secteur d'activités qui perdra le plus d'effectifs d'ici 2020 (Ibid.). Étudier la durabilité des activités agricoles dans les territoires demande d'articuler plusieurs échelles non seulement temporelles mais aussi spatiales (l'exploitation, le tissu d'exploitation) (Gibon et al., 2010). Comment dure et évolue une exploitation au cours d'une carrière professionnelle et au-delà de la carrière lors des transmissions (Gibon 2003; Mottet et al., 2006 ; Gibon et al., 2010) ?

Dans les zones de montagne, la transmission des exploitations prend une dimension particulière. L'agriculture et l'élevage jouent un rôle central dans le maintien de paysages ouverts fournissant une plus grande diversité de fonctions que les paysages couverts par la forêt : biodiversité, limitation des risques d'incendies, valeur esthétique, aménités et valorisation agricole du territoire. Dans ces zones, il est donc crucial de gérer les problèmes liés au maintien d'un tissu d'exploitations sur un

1. Groupe ESA, Unité de recherche sur les Systèmes d'élevage, 49007 Angers, France ; INRA, UMR 1201 Dynafor, 31326 Castanet Tolosan cedex, France, c.manoli@groupe-esa.com.

2. INRA, UMR 1201 Dynafor, 31326 Castanet Tolosan cedex, France, jean-philippe.choisis@toulouse.inra.fr.

3. INRA, UMR 1201 Dynafor, 31326 Castanet Tolosan cedex, France, sylvie.ladet@toulouse.inra.fr.

4. INRA, UMR 1201 Dynafor, 31326 Castanet Tolosan cedex, annick.gibon@toulouse.inra.fr.

Ce chapitre de l'ouvrage L'agriculture en famille : travailler, réinventer, transmettre est publié en Open Access sous licence creative commons CC-BY-NC-ND permettant l'utilisation non commerciale, la distribution, la reproduction 
territoire donné. Par ailleurs, et particulièrement dans les zones touristiques, l'utilisation de l'espace et donc l'acquisition de foncier sont soumises à la concurrence entre plusieurs activités : tourisme, agriculture, habitat... une concurrence d'autant plus forte que les zones les plus favorables à toutes ces activités sont peu étendues (par exemple terres de fond de vallée ou de mi-vallée).

L'analyse de l'évolution des exploitations sur le long terme a donné lieu au développement en sciences agronomiques de la notion de "trajectoires d'exploitation » et de méthodes spécifiques à leur approche via l'étude des modifications de la structure et du fonctionnement des exploitations (Capillon, 1993 ; Gibon et al., 1999 ; Moulin et al., 2004). La dimension temporelle considérée a généralement été la carrière professionnelle de l'exploitant, de son installation à son départ en retraite. Pourtant, l'étude des exploitations et des activités agricoles dans les territoires amène à accorder un intérêt particulier à la place de la transmission dans ces trajectoires. La transmission y apparait comme un moment charnière. Les travaux sur l'évolution de l'élevage dans les Pyrénées (Mottet, 2005 ; Mottet et al., 2006 ; Rueff et al., 2012) et dans les coteaux de Gascogne (Ryschawy et al., 2013 et dans ce même ouvrage), montrent ainsi que les recherches sur les transformations des activités agricoles et de la gestion de l'espace à l'échelle du territoire imposent de considérer les trajectoires des exploitations selon une échelle temporelle qui dépasse la carrière professionnelle de l'exploitant, et de développer des méthodes pour cela.

La recherche, dont nous rendons compte ici, s'inscrit dans les travaux de modélisation des trajectoires des exploitations sur des pas de temps qui vont au-delà de la trajectoire professionnelle d'un exploitant. Nous avons développé une approche intégrée de l'étape que constitue la transmission dans la trajectoire de l'exploitation. La transmission des exploitations est ici vue comme une étape particulière de l'évolution au long cours des «systèmes famille-exploitation " (par ex. Osty, 1978). Notre étude s'appuie sur des avancées des sciences sociales (Bortoli, 2007) et un ensemble d'études de cas dans la région des montagnes de Bigorre.

De par cette orientation, le travail exploratoire mené s'inscrit dans l'ensemble des travaux qui visent à éclairer la compréhension des logiques d'action des agriculteurs sur le long terme en vue de conforter la résilience des exploitations (Dedieu et al., 2008). Nous y reviendrons dans la conclusion.

Après avoir exposé la problématique et la méthode de recherche suivie, nous présenterons un modèle générique de l'exploitation agricole adapté à la prise en compte de la transmission intergénérationnelle. Nous tenterons enfin d'éclairer, à la lueur de ce modèle, la diversité des modalités de transmission de l'exploitation dans un échantillon de douze exploitations des montagnes de Bigorre. 


\section{Problématique et méthode de recherche}

\section{Positionnement}

Dans les travaux présentés ici, nous avons souhaité articuler différents points de vue disciplinaires développés sur la transmission des activités, du foncier, du capital. En développant une analyse intégrée de la dynamique des activités d'élevage dans les territoires (Gibon et Hermansen, 2006), nous abordons la pérennité de l'exploitation sous l'angle " socio-technique » et, dans une moindre mesure, "socioécologique ", en accordant un intérêt particulier aux pratiques des exploitants et aux déterminants sociaux de ces pratiques.

Nous avons travaillé à l'échelle de l'exploitation. Au niveau temporel, nous nous sommes intéressés à l'évolution de long terme des exploitations, sur une période qui couvre plus de 50 ans, avec la succession de plusieurs générations.

\section{Démarche générale}

Les démarches d'étude intégrée des systèmes agraires et des exploitations agricoles se fondent depuis longtemps sur les théories relatives à la modélisation des systèmes complexes à l'interface entre nature et société (Jollivet, 1992 ; Legay, 1996) et adaptées à l'articulation de points de vue disciplinaires (Delattre, 1995). Elles reposent sur des approches itératives associant raisonnements inductif et déductif, avec des allers-retours entre des données empiriques issues du terrain et des données théoriques issues de la bibliographie. Nous avons appliqué cette démarche exploratoire et itérative afin de relier les changements structurels et fonctionnels des exploitations aux comportements de la famille au cours et au-delà du cycle de vie. Nous détaillons ici les différentes étapes de la démarche.

\section{La mobilisation des cadres théoriques de la modélisation des systèmes complexes}

Pour construire une approche intégrée, nous avons mobilisé les principes de la modélisation des systèmes complexes dits pilotés (entreprise, exploitation agricole ou autre), présentés dans les travaux de Le Moigne (1994) et Mélése (1991). L'exploitation est vue ici comme un système "famille/exploitation " : la famille et ses activités sont considérées comme faisant partie du système étudié (Osty, 1978). Au sein du système famille/exploitation, c'est tout particulièrement le " pilote " qui nous intéresse ; ce pilote n'est pas ici le seul chef d'exploitation, mais c'est le collectif familial (Ibid.). Pour étudier l'étape de transmission des exploitations, nous nous sommes tout particulièrement attachés aux " logiques " de ce pilote, en référence à Mélèse (1991) qui traite du pilotage stratégique des organisations. Cet auteur définit les "logiques " comme des "champs " transversaux qui coexistent dans le pilotage à long terme de l'organisation considérée. Nous avons donc formulé l'hypothèse que dans la gestion de la transmission de l'exploitation coexistent chez 
les familles agricoles plusieurs « logiques " qui correspondent à différentes façons de voir l'évolution de l'exploitation dans ses relations à son environnement. Dans ce travail, notre objectif a été d'identifier et décrire ces logiques à partir de cas réels d'exploitations.

\section{En agronomie, l'approche " globale " des dynamiques d'exploitation sur le long terme}

L'analyse des dynamiques d'évolution des exploitations sur le long terme (les trajectoires de changement des exploitations) a fait l'objet de divers travaux de recherche en agronomie sensu lato (Perrot, 1990 ; Capillon, 1993 ; Gibon et al., 1999; Mottet, 2005 ; Moulin et al., 2008 ; Cialdella et al., 2009 ; Rueff et al., 2012 ; Ryschawy et al., 2013). Ces trajectoires y apparaissent comme un ensemble d'étapes jalonnées par des changements. Selon Moulin et al. (2008), ces changements sont liés à des facteurs externes ou internes au système famille-exploitation, et peuvent être soit majeurs, car ils affectent alors la cohérence du système, soit mineurs, car ils n'entraînent alors pas de changement de cohérence. Dans la plupart de ces travaux, la transmission est vue comme une phase de rupture franche. Tout se passe alors comme si une exploitation à la génération $n$ pouvait être considérée comme une entité différente de l'exploitation à la génération $n+1$. Notre hypothèse est qu'il existe non seulement des éléments de rupture mais aussi des éléments de continuité dans le processus de transmission. Notre objectif est d'élaborer une vision et une méthode d'approche de la transmission, dans la dynamique globale de l'exploitation, qui permette de prendre en compte et de décrire ce qui fait continuité et ce qui fait rupture dans un système famille exploitation, au moment de la transmission.

\section{Un recours à des concepts et méthodes issus d'autres disciplines pour modéliser la transmission}

Différents points de vue sur la transmission des exploitations ou des entreprises, développés dans le champ des sciences sociales, sont mobilisés ici. Pour Gasson et Errington (1993), qui l'ont étudiée d'un point de vue socio-économique, la transmission des exploitations agricoles est un processus de négociation entre générations montante et descendante. Ce processus est composé de plusieurs étapes, dont notamment le transfert de gestion et de propriété. Mignon (2001) et Macombe (2006), en sciences de la gestion, ont, quant à elles, étudié les questions de transmission des entreprises (non agricoles et agricoles respectivement) en référence à la notion de pérennité. Selon ces auteurs, la pérennité d'une entreprise repose sur un transfert du capital, de la direction, des activités, mais elle repose aussi sur l'existence d'une "identité de l'organisation " (Mignon, 2001), sur la transmission de valeurs de l'entreprise (une "éthique du métier", (Macombe, 2006)).

Dans le cas de la transmission des exploitations familiales en milieu agricole pyrénéen et dans le Sud-Ouest de la France en général, ces notions de valeurs et d'identité sont reprises dans les travaux d'ethnologues et d'anthropologues portant sur les communautés rurales organisées selon un principe de "système à maison " 
(Lamaison, 1987). La " maison ", " domaine composé de biens matériels et immatériels " (ibid) est, en effet, le cadre traditionnel de l'exploitation agricole dans le SudOuest (Augustins, 1977 ; de Bortoli, 2007 ; Sourdril, 2008) ; ce qui implique de la transmettre intégralement entre les générations ; devant elle, l'intérêt de chaque individu pris isolément doit s'effacer. Ce cadre traditionnel de transmission familiale évolue aujourd'hui, notamment dans un contexte où le foncier et l'immobilier prennent une valeur monétaire importante en montagne.

Ces différents points de vue issus de la littérature ont contribué à l'identification des grandes « logiques » de pilotage stratégique des exploitations sur le long terme dans les montagnes de Bigorre.

\section{Saisir et comprendre la diversité des modalités et des enjeux de la transmission des exploitations}

\section{Le choix de " clés d'entrée " sur le sujet}

Le choix de "clés d'entrée " dans l'étude de systèmes complexes (par ex., Gibon, 2003) revient à privilégier, dans le recueil et le traitement des données, certains axes d'analyse, dont on fait l'hypothèse qu'ils sont des facteurs importants pour comprendre la diversité de structure et de dynamique des systèmes étudiés. Pour saisir la diversité des processus de transmission des exploitations dans notre zone d'étude, nous avons retenu trois clés d'entrée : la pluriactivité qui est vue comme un facteur important d'explication de la durabilité des exploitations (Ponchelet et Bellon, 1999 ; Laurent et Rémy, 2000 ; Cialdella, 2005) ; le système de la " maison " pyrénéenne dans lequel peuvent s'inscrire encore (ou pas) les exploitations (de Bortoli, 2007 ; Sourdril, 2008) ; la transmission vue non pas comme un moment spécifique mais comme un processus composé de plusieurs étapes (Gasson et Errington, 1993). Ces clés d'entrée ont servi au choix de l'échantillon et à la construction du questionnaire ; l'objectif étant de saisir les variations existantes, autour de ces trois thèmes, dans la zone d'étude.

\section{Dispositif d'enquête : un échantillonnage " en entonnoir "}

Nous nous sommes appuyés sur les résultats d'une enquête de 2003 auprès de l'ensemble des agriculteurs exploitant des terres sur quatre communes du "Davantaygue " (montagnes de Bigorre, département des Hautes-Pyrénées, zone d'adhésion du parc national des Pyrénées). Ces quatre communes sont situées dans une zone de moyenne montagne, assez touristique. Cette enquête a été menée dans le cadre d'un travail de modélisation multiéchelles des interactions entre agriculture et paysage (Gibon et al., 2010). Nous avons mobilisé les résultats relatifs à la diversité de la structure, du fonctionnement et de l'évolution de ces quarante exploitations ainsi que les données sur l'histoire et la composition des familles depuis les années 1950 (Mottet, 2005). Une exploitation complémentaire de ces données a permis de caractériser les exploitations selon la pluriactivité des membres de la famille, le 
moment dans le cycle de vie des exploitations, l'existence ou non de transmissions correspondant au modèle de la " maison " afin de construire un échantillon stratifié en référence aux trois clefs d'entrée précédemment évoquées. Douze exploitations sur les quarante ont été sélectionnées pour constituer un échantillon d'exploitations diversifiées au regard de ces différents critères (Manoli, 2008).

Des entretiens complémentaires ont été menés dans les douze exploitations de l'échantillon. Ils ont été réalisés avec les chefs d'exploitation en présence des conjoints ou d'autres membres de la famille (parents, enfants) quand cela était possible. Ces entretiens, de type semi-directif (par ex. Kaufmann, 2001), ont porté sur les changements survenus dans le système famille/exploitation depuis l'enquête du printemps 2003, et sur le détail du processus de transmission de l'exploitation au cours du (ou des deux) transfert(s) intergénérationnel(s) intervenu(s), selon les cas, depuis 1950 .

Sur deux des douze exploitations enquêtées, une étude monographique des processus de modification du parcellaire de l'exploitation a été réalisée afin d'avoir un énoncé fin des événements liés à l'évolution et à la transmission du foncier depuis 1950. Dans cette approche monographique, des cartes du parcellaire cadastral des deux exploitations ont servi de média pour l'accès à l'information (Sourdril et Ladet, 2008). Elles ont permis d'aborder de façon plus approfondie, que pour les dix autres cas, le sujet de la transmission et les changements liés au parcellaire survenus depuis les années 1950. La figure 2 présente l'un de ces cas approfondis. Les deux cas retenus pour cette étude ont été choisis en raison des différences fortes qu'ils présentaient dans les modalités de transmission de l'exploitation : une transmission présentant des éléments forts de rupture, tandis que l'autre montrait davantage de continuité. De plus, les familles à la tête de ces deux exploitations avaient montré un intérêt fort pour le thème de la transmission. Celle-ci était en cours dans l'une, et l'autre exploitation avait traversé cette étape peu de temps auparavant.

Ces enquêtes ont été complétées par des entretiens avec d'autres acteurs locaux, disposant d'une expertise sur les questions de transmission des exploitations dans la région d'étude. L'objectif était de disposer d'un témoignage complémentaire à celui des exploitants afin d'éclairer des points en apparence contradictoires soulevés par ces derniers. Des entretiens d'une durée de une à deux heures ont été effectués avec :

- un technicien de la Chambre départementale d'agriculture des Hautes-Pyrénées, afin de mieux cibler les enjeux sur la zone avant le démarrage des enquêtes en exploitation ;

- un responsable de l'ADASEA ${ }^{5}$ du département, pour avoir une discussion sur le déroulement de transmissions en cours sur la zone ;

- le notaire d'Argelès-Gazost, la ville la plus proche des quatre communes du Davantaygue, qui a permis de mieux comprendre la juridiction en vigueur et les écarts de pratiques par rapport à la loi (prêts à commodat, soulte par exemple).

5. Association départementale pour l'aménagement des structures des exploitations agricoles. 


\section{Trois « logiques » de pilotage stratégique des exploitations}

"On essaye que ce (l'exploitation) soit dans la famille et (que ça reste) agricole. " Chef de l'exploitation agricole $\mathrm{n}^{\circ} 7$.

La bibliographie et les études de cas nous ont conduits à développer un modèle qui repose sur un découpage modulaire de l'exploitation selon trois grandes logiques de pilotage stratégique (Fig. 1). Ce modèle représente en fait leur coexistence.

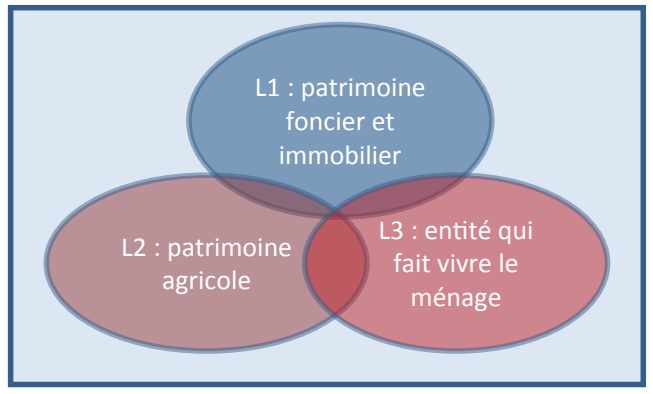

Fig.1. Les trois logiques de pilotage stratégique de l'exploitation agricole (EA) dans les processus de transmission intergénérationnelle en montagne pyrénéenne.

Dans une première logique L1, l'exploitation est vue comme un patrimoine doté d'une valeur foncière et immobilière. L'exploitation est en effet un ensemble de biens fonciers et immobiliers qu'il faut gérer, accroître, valoriser mais aussi penser à léguer à un ou plusieurs enfants.

Une deuxième logique L2 est celle où l'exploitation est vue comme un patrimoine agricole matériel et immatériel. L'exploitation est un ensemble d'activités agricoles qui se déroulent au quotidien. Elles permettent l'entretien du territoire et donc le maintien de la valeur d'usage agricole des terres et des agroécosystèmes qui les occupent (prairies semi-naturelles). L'exploitation est un lieu d'élaboration et de valorisation d'une production au moyen de savoirs et savoir-faire socio-techniques. Toutes ces activités ont un sens particulier pour l'exploitant. Elles doivent pouvoir se perpétuer et se transformer sur le long terme pour assurer la pérennité de l'exploitation.

Enfin, la troisième logique L3 est celle où l'exploitation est vue comme une entreprise qui contribue à faire vivre le ménage. Celui-ci peut assurer ses moyens d'existence par un système d'activités (Laurent et Rémy, 2000) incluant diverses tâches professionnelles et d'autres sources de revenu (pensions). À ce " faire vivre " le ménage, l'exploitation contribue quantitativement de presque rien dans les exploitations pluriactives qui ont une activité extérieure à temps plein, à beaucoup quand le revenu agricole permet de faire vivre l'intégralité de la famille. Cette logique est celle du " quotidien » et constitue une dimension essentielle pour penser la transmission à la génération suivante. 
Dans la suite des résultats, nous allons décrire les enjeux, les contraintes et la diversité des pratiques s'exprimant, sur le terrain d'étude, autour de ces trois grandes logiques de l'exploitation.

\section{Première logique (L1) : transmettre un patrimoine foncier et immobilier}

\section{Une sécurisation progressive et graduelle du foncier}

L'accès au foncier s'est révélé être une préoccupation constante pour la majorité des exploitants enquêtés. Les exploitants et les experts ont souligné l'augmentation importante, depuis les années 1950, du pourcentage de terres en location dans la surface agricole utile (SAU), et particulièrement des terres en location verbale non déclarée ( $c f$. le cas de l'exploitation présentée en figure 2).

L'acquisition de foncier supplémentaire est apparue comme un objectif majeur, se déroulant fréquemment en plusieurs étapes avant d'aboutir à la propriété. L'étude de cas cartographique (Fig. 2) illustre le déroulement de ces étapes : pour de nombreuses parcelles, l'exploitant commence par utiliser des terres en location verbale non déclarée (par exemple, parce que le propriétaire arrête son activité agricole) ; puis après quelques années, il réussit à la transformer en une location verbale déclarée auprès de la mutuelle sociale agricole (MSA), et à avoir des droits sur la terre plus importants : accès aux aides agricoles ; droits prioritaires en cas de vente. Enfin, il achète dès que l'occasion se présente. En fait, ces trois étapes (locations non déclarées puis déclarées enfin propriété) apparaissent comme des gradations dans la sécurisation du foncier. Les études de cas cartographiques révèlent aussi que l'accès à de nouvelles terres agricoles ou constructibles est recherché en permanence, tout au long du cycle de vie d'un exploitant, en lien avec l'objectif de renforcer la «transmissibilité » de l'exploitation. La phase d'installation du repreneur, n'est pas le moment crucial de cette sécurisation. Au contraire, elle apparaît souvent plutôt comme un moment de vulnérabilité. Notamment, lors de l'installation d'un jeune, la justification de la surface minimale requise de terres exploitées pour obtenir des aides à l'installation pose de plus en plus souvent problème, aux dires des exploitants et des experts consultés, tant le pourcentage de terres en location non déclarée devient important dans les exploitations ${ }^{6}$.

\section{Des enjeux forts sur le foncier}

Cette stratégie d'accès graduel au foncier s'explique en partie par le contexte de la zone, dont le fort potentiel touristique entraîne de très fortes concurrences pour l'accès à la propriété du foncier bâti et non bâti. Les granges foraines situées en zone intermédiaire ou de haute montagne sont ainsi devenues très convoitées pour servir

6. Leur proportion moyenne était de $30 \%$ en 2003 selon les résultats de l'enquête (Gibon et al., 2010). 
de résidences secondaires. Dans ces zones, les terres ne sont pas constructibles, mais l'utilisation de ces granges à des fins d'habitation est autorisée à certains endroits. Leur acquisition par une population non agricole, d'origine urbaine ou étrangère, a conduit à une hausse des prix de vente considérable pour la région. Le phénomène spéculatif sur les parcelles qui hébergent des granges foraines rend l'accès à la propriété de ces parcelles difficile pour un agriculteur, car ces parcelles atteignent des prix sans relation avec leur potentiel productif. Un phénomène similaire touche les terres à fort potentiel agricole autour des villages, convoitées pour la construction immobilière. Notons que, paradoxalement, des agriculteurs peuvent être euxmêmes acteurs et bénéficiaires de cette spéculation foncière quand ils vendent des granges foraines à des prix bien supérieurs à ceux estimés lors des successions familiales, afin d'assurer le financement de la construction de bâtiments agricoles ou d'autres investissements sur l'exploitation.

La location verbale non déclarée de parcelles, avec ou sans granges foraines, s'est donc fortement développée au sein du territoire des exploitations. En effet, cette location verbale non déclarée n'assure pas, en cas de vente, le droit à la propriété préférentielle à l'agriculteur qui exploite ces parcelles (droit de préemption), et est de ce fait préférée par les propriétaires. C'est donc non seulement l'accès à la propriété qui est rendu difficile, mais aussi l'accès à la location déclarée. Comme nous l'avons indiqué, la non-déclaration des terres en location pose un problème sérieux lors de l'installation des jeunes et fragilise la pérennité des exploitations : le propriétaire des terres peut décider de vendre à tout moment. Cette précarité de l'accès à ces surfaces peut avoir pour conséquence que les agriculteurs y assurent moins d'investissements qu'ailleurs (travaux de déboisement ou d'aménagement des chemins d'accès par exemple).

À cette concurrence pour l'accès à la propriété des parcelles où se situent les granges, s'ajoute une concurrence entre agriculteurs pour l'accès à la location. Elle concerne surtout les terres plates situées en fond de vallée, les plus rares et les seules facilement mécanisables.

Une des solutions trouvées aux problèmes de sécurisation de l'accès à la terre est d'exploiter des parcelles dans d'autres vallées. Plusieurs exploitations enquêtées ont suivi cette voie. L'exemple traité dans l'analyse cartographique l'illustre : cette famille a pris en fermage deux hectares dans une commune avoisinante en $1998^{7}$ (Fig. 2).

\section{Problèmes posés par le besoin de sécurisation du foncier}

Une solution " adaptée " pour l'accès au foncier des agriculteurs, exposée par les experts locaux, sont les baux nommés prêts à commodat. Ce sont des « baux aménagés " à court terme, des "baux dérogatoires au statut de fermage " (cf. communication

7. Les terres en fermage appartenaient à une même famille agricole cessant son activité. À l'occasion d'une enquête ultérieure dans la région (Bailhe, 2010), un membre de cette famille nous a confirmé que quelques terres en fermage avaient été cédées à deux jeunes « qui en avaient besoin " alors que le restant était repris par un cousin. 
avec le notaire). Ils sont signés pour une période de cinq ans puis tacitement renouvelables tous les ans. Après la première période de cinq ans, ces baux ne garantissent donc qu'annuellement à l'agriculteur un accès prioritaire aux ressources. Présentés comme " la solution idéale " par le notaire, ils sont ignorés des agriculteurs qui préferent exploiter des terres situées à plus de $20 \mathrm{~km}$ de distance du siège de l'exploitation mais mieux sécurisées (Fig. 2, "État des lieux en 1998 »). Selon nous, l'adoption de baux à court terme ne satisfait pas le besoin de sécurisation à long terme exprimé par les agriculteurs. Cela confirme qu'au-delà d'un problème d'accès aux ressources, il y a surtout pour les exploitants un problème de sécurisation sur le long terme de l'accès aux parcelles. Ce qui permettrait d'expliquer un autre paradoxe souligné par le notaire, les conseillers et les agriculteurs eux-mêmes : alors même que la compétition pour l'accès au foncier est forte, il existe de nombreuses parcelles non exploitées, ou sous exploitées car elles sont l'objet d'un partage familial en cours. Non seulement ces parcelles sont parfois les plus difficilement exploitables (accès, fertilité...), mais il est aussi impossible pour les agriculteurs d'investir à long terme sur ces parcelles (entretien des chemins d'accès, défrichage...). En effet, en l'absence de connaissances sur qui sera le propriétaire à venir et donc le devenir des parcelles, ces investissements n'offrent aucune rentabilité.

\section{Deuxième logique (L2) : Transmettre un patrimoine agricole}

\section{Installation et transmission : des processus plutôt que des dates}

Installer un repreneur était toujours présenté dans les entretiens comme un processus et non comme un moment se résumant à la date officielle d'installation : "cest très progressif ", "c'est sur le tas ", " on ne se rend compte de rien "... Les transferts de compétences et de responsabilités semblent ainsi se faire de façon très progressive avec, par exemple, la délégation préalable de tâches techniques. En amont, la transmission se prépare chez la génération cédante par une réflexion sur le choix du successeur parmi les enfants : lequel a le plus de goût pour les activités d'élevage par exemple ? Lequel a le conjoint le plus motivé ou avec la situation professionnelle la plus compatible pour la reprise ? En fait, la transmission peut être décomposée en un ensemble de phases chronologiques : le transfert de gestion, le transfert de propriété et la date officielle de changement de nom du chef d'exploitation (CE).

\section{Distinguer les notions de chef d'exploitation et de pilote}

Il peut y avoir décalage entre le chef d'exploitation "officiel » et le pilote effectif de l'exploitation, que l'on peut qualifier de chef d'exploitation « fonctionnel » (Mottet, 2005). Au cours des entretiens, les dates d'installation des chefs d'exploitation successifs étaient toujours des références connues, mais lors des questions sur la gestion de l'exploitation, la notion de " prête-nom » vis-à-vis de l'administration transparaissait. Un prête-nom est en fait quelqu'un qui a été choisi, par arrangement familial, parmi le couple, les parents, les enfants ou les gendres et belles-filles, pour avoir le statut de chef d'exploitation et accéder aux avantages qui lui sont liés. 
Dans les douze études de cas, le pilote effectif de l'exploitation apparaissait, de plus, rarement comme un individu seul, mais plutôt comme un collectif familial, parfois multi-générationnel, constitué d'un gestionnaire principal, accompagné de co-décisionnaires. Lorsque le chef d'exploitation déclaré n'est pas le plus investi dans le pilotage de l'exploitation, nous le qualifierons dans la suite du texte de "prêtenom ", reprenant en cela la terminologie utilisée par les agriculteurs. Ce terme n'est donc pas à prendre au pied de la lettre comme désignant le représentant d'un autre individu, mais au sens élargi de représentant du collectif décisionnel familial. Ce prête-nom est le plus souvent choisi pour des raisons économiques (opportunité d'accès aux aides, à la retraite agricole, aux prêts pour l'installation). Il peut être un fils ou une épouse comme une belle-fille ou un neveu. Ce prête-nom peut remplir un rôle réel dans la gestion de l'exploitation : travail quotidien ou occasionnel, participation aux décisions, investissement progressif. Mais ce peut être aussi quelqu'un qui n'a pas ou n'a plus aucun rôle dans cette gestion : femme d'exploitant qui n'a jamais été intéressée par l'exploitation ou exploitant à la retraite...

Les dates administratives de changement de nom du chef d'exploitation officiel correspondent donc très rarement à un transfert effectif de la gestion de l'entreprise agricole. L'installation officielle d'un jeune peut ainsi se faire avec un maintien complet des prises de décision dans l'exploitation par le cédant, censé être à la retraite.

\section{Troisième logique (L3) : transmettre une entreprise agricole}

\section{Un usage " opportuniste » de sources de revenus diversifiées}

Transmettre une exploitation agricole, c'est transmettre une source de revenus pour la famille. Le plus souvent, les revenus apportés par la seule exploitation agricole ne suffisent pas à eux seuls à faire vivre la famille, compte tenu de la taille des exploitations et des systèmes de production extensifs pratiqués (Mottet, 2005). La très grande majorité des familles étudiées " jongle " entre les différentes sources de revenus auxquelles elles ont accès pour être capables d'assurer le maintien sur le long terme, et en continu, d'une exploitation.

Les familles semblent ici pour la plupart raisonner l'avenir et la pérennité de leur exploitation non pas en considérant globalement le revenu que peuvent générer les activités agricoles (produits et aides assurées par les dispositifs publics liés à l'agriculture et l'agrienvironnement) et celui que peuvent apporter des activités professionnelles extérieures mais aussi en prenant en compte globalement l'ensemble des moyens économiques à la disposition du ménage (en particulier les retraites, les possibilités de vendre une grange ou un terrain à bâtir pour faire face à un besoin spécifique...). Ces stratégies peuvent apparaitre comme relevant d'une forme d'opportunisme où un ensemble de ressources variées est mobilisé pour constituer le revenu nécessaire sur le court-terme et assurer la pérennité à long terme de l'exploitation agricole. La façon dont les familles mobilisent les dispositifs d'aide à l'agriculture, en plus des formes de recours à la pluriactivité, illustre bien cette logique. 


\section{Des jeux de " prête-nom " pour tirer bénéfice des aides à l'agriculture}

Le choix du " prête-nom » est un jeu subtil qui peut être mis en relation avec la mobilisation des aides en agriculture. Il est souvent basé sur le statut de chaque membre de la famille : telle belle-fille est par exemple nommée chef d'exploitation parce que son beau-père (qui en restera le gestionnaire), peut profiter de ses droits de retraite et parce qu'elle ne travaille pas à l'extérieur, contrairement à son mari et aux frères ou sœurs de son mari. Cette personne peut également être choisie si elle peut bénéficier des aides à l'installation. Le choix du prête-nom est basé sur des arguments d'opportunités économiques : quel meilleur profit peut-on tirer, à un moment donné, d'un système d'aides et de pension ? Cette stratégie est familiale : pour ce calcul, on prend en compte les statuts et les disponibilités de tous les membres de la famille. Mais il serait faux de considérer cet opportunisme comme une course systématique aux aides et aux primes. Dans de nombreux cas, les familles ont préféré renoncer à des aides pour financer un investissement, et attendre de pouvoir l'autofinancer plutôt que de modifier une conduite de l'exploitation jugée « inadaptée aux conditions du milieu » (Bailhe, 2010).

\section{La pluriactivité comme facteur de pérennité à long terme des exploitations}

Présentée d'abord par les agriculteurs comme un danger pour les exploitations, la pluriactivité apparaît comme une opportunité pour la majorité des exploitations de se maintenir. De fait, sur les 50 ans écoulés, certains chefs d'exploitation ont travaillé à l'extérieur puis sont revenus à l'activité agricole. Si on considère la totalité du collectif familial (époux [ses], enfants) toutes les familles enquêtées ont en fait connu des phases de pluriactivité, au cours des 50 dernières années. La grande majorité de nos interlocuteurs voit cela comme une source de difficultés pour la gestion de l'activité agricole, du fait de la contrainte de temps de travail lié au cumul des deux activités. La mise en place des 35 heures est d'ailleurs un avantage fort qui a permis, en libérant du temps pour les salariés, de conjuguer plus aisément deux métiers :

"les 35h, ça a relancé les exploitations. " (Belle-mère du chef de l'exploitation agricole $\mathrm{n}^{\circ} 7$, avril 2008).

La pluriactivité est une source de moyens financiers pour investir dans les exploitations, et dans des innovations techniques qui allègent le travail. Un exemple particulièrement illustratif nous est donné par l'histoire du père de l'un des chefs d'exploitation rencontrés :

"Mon père, il a travaillé 26 ans à la Cophase (usine chimique), il a arrêté en 1974/1976 car il y avait des grèves, pendant six mois. Il a monté le projet du " horssol", il a monté les bâtiments au lieu de rester à rien faire. Il a commencé le 16 juin, en octobre il y avait 300 veaux dans le bâtiment. Le partage de famille avait eu lieu en 1970/1971: il a continué la Cophase, il faisait les deux. Puis en 1976, quand il a construit le bâtiment et il n'a fait plus que ça, il n'a pas repris après la grève. " (Chef de l'exploitation agricole $\mathrm{n}^{\circ} 11$, avril 2008). 
Dans ce cas, la pluriactivité apparaît comme un véritable tremplin pour revenir à une activité uniquement agricole : l'argent gagné en usine, le temps laissé par un événement exceptionnel (la grève) ont été réinvestis dans l'exploitation. La pluriactivité représente donc pour les agriculteurs enquêtés un moyen de maintenir sur le long terme l'exploitation familiale.

\section{Une illustration de l'imbrication de ces trois logiques sur le long terme}

Cette analyse des trois logiques d'action permet de faire émerger trois types de stratégies mises en place par les exploitants pour durer : la sécurisation progressive du foncier de l'exploitation ; le partage multi-générationnel des rôles et de la gestion de l'exploitation ; l'appui opportuniste du collectif de travail familial sur des sources de revenus extérieures.

L'analyse cartographique des évolutions sur le long terme menée sur deux cas contrastés a permis de mieux comprendre comment ces différentes logiques et stratégies se sont imbriquées sur le long terme. Ces deux cas illustratifs montraient deux façons de transmettre l'exploitation agricole : une transmission de type « continuité ", développée dans l'encadré 1, et une transmission qui s'est faite beaucoup plus en rupture.

Encadré 1. Un cas de transmission d'exploitation de type " continuité », vue à travers l'évolution de son parcellaire.

Le cas présenté en figure 2 correspond à une transmission de type " continuité ". L'évolution de l'exploitation est vue à travers l'évolution de son parcellaire. Les dates qui rythment cette description sont les dates pour lesquelles il y a eu des changements importants du parcellaire, du gestionnaire principal, ou des évènements familiaux qui ont changé l'organisation du collectif de travail. Cette trajectoire est marquée par le fait qu'elle a été très graduelle, les différentes générations ont été impliquées progressivement dans la vie de l'exploitation. Paul ${ }^{8}$ (génération $n+1$ ) et son fils JeanPhilippe (génération $n+2$ ) élèvent des espèces différentes (des bovins pour le premier et des ovins pour le second), ce qui permet aux deux générations d'avoir chacun son activité sur une même exploitation. L'autonomie de la jeune génération est ainsi compatible avec l'autorité de la génération antérieure. On peut supposer que l'apprentissage entre les deux générations est facilité pendant cette période de co-gestion de l'exploitation.

8. Les prénoms ont été changés pour des raisons de confidentialité. 


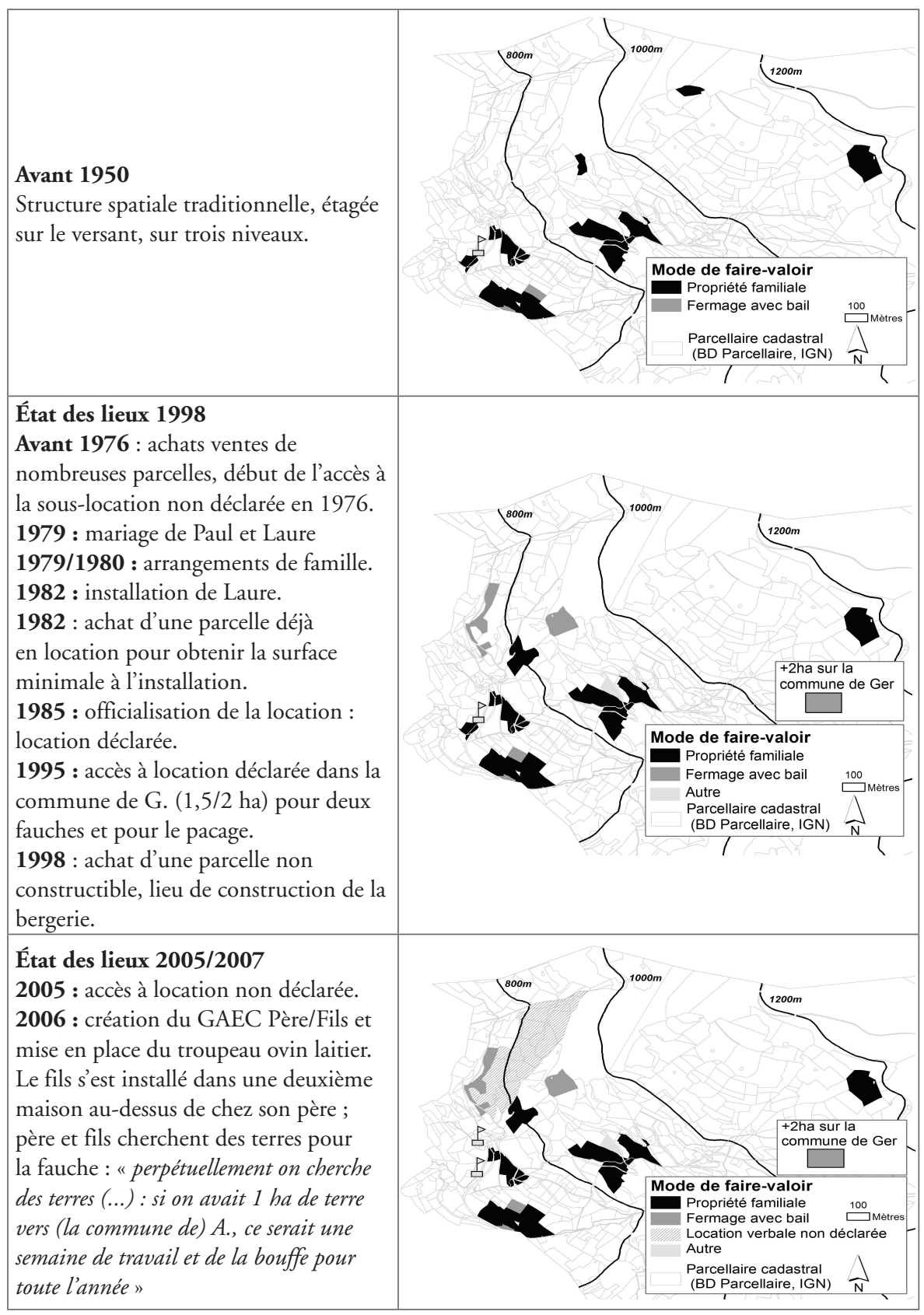

Fig. 2. Évolution parcellaire depuis les années 50 de l'exploitation de Paul, Laure et leur fils Jean-Philippe (diffusé avec l'autorisation des agriculteurs). Les années considérées sont illustratives des différentes phases d'évolution de l'exploitation. Les drapeaux représentent les sièges d'exploitation. 
Dans ce deuxième cas, en rupture, la transmission officielle de l'entreprise agricole, en cours lors de notre passage, se heurtait à de fortes difficultés. L'autorité exercée par le père (génération $n+1$ ) sur son fils (génération $n+2$ ) en train de s'installer était très forte. Aux dires du père (génération $n+1$ ), son père (génération $n$ ) très autoritaire, avait conservé toute autorité sur la gestion de son exploitation jusqu'à sa mort, même après sa retraite "officielle ». Dans ce second cas, les transmissions entre générations se sont faites de manière soudaine, le repreneur ayant été peu préparé à la prise en charge des décisions de gestion de l'exploitation. De plus, le moment de la reprise se retrouvait être un moment où il fallait aussi régler des conflits de famille, pour des histoires de partage de foncier et d'héritage. Le père (génération $n+1$ ) est un pluriactif, le jeune qui reprend souhaite avoir seulement une activité agricole. La reprise par le jeune avait donc lieu dans des conditions où il cumulait les difficultés.

Dans l'autre cas, celui où la transmission s'était déroulée plus en continuité (encadré 1), on observe la présence des mêmes stratégies et logiques que dans le cas en rupture, en revanche, la transmission se fait avec beaucoup moins de heurts : transfert de gestion, implication dans les statuts de l'exploitation se font de façon progressive. Les discussions pendant les entretiens sur le sujet de la transmission montraient qu'elle était beaucoup moins vécue comme un point sensible et difficile que dans la trajectoire en rupture. La pluriactivité, dans ce cas d'exploitation, concerne la femme du chef d'exploitation.

Ces deux exemples illustratifs nous montrent donc que les trois logiques et les différentes stratégies sont présentes mais elles s’imbriquent différemment et il s'ensuit des phases de transmission qui se déroulent avec plus ou moins de ruptures, de continuités entre les générations.

\section{Discussion}

\section{La méthode et les outils}

La difficulté de l'étude socio-technique des processus de transmission intergénérationnelle des exploitations agricoles fait qu'ils ont été peu étudiés jusqu'ici en sciences agronomiques. La démarche exploratoire adoptée est faite d'itérations entre la mobilisation des bases théoriques et méthodologiques d'étude des systèmes famille-exploitation en sciences agronomiques et en sciences sociales, et de la réalisation d'enquêtes socio-techniques de terrain.

Pour la méthode mise en place, l'utilisation de cartes SIG comme supports de médiation dans les entretiens avec les agriculteurs s'avère particulièrement féconde pour accéder à des données de qualité (Mottet, 2005 ; Sourdril et Ladet, 2008). L'utilisation de cartes du parcellaire de l'exploitation à différentes dates a été précieuse pour pouvoir comprendre les évolutions et stratégies liées au foncier de 1950 à 2008. La discussion à partir de ces cartes nous a permis de retracer l'évolution du foncier, mais aussi de discuter en détail la manière dont les exploitants se représentent les différents événements survenus au fil des ans. 


\section{Une étude du collectif familial de gestion de l'exploitation agricole}

Les résultats de notre étude soulignent le rôle du collectif familial dans la gestion sur le long terme des exploitations. Alors que le collectif familial est classiquement perçu comme un pôle de gestion unique dans les études agronomiques du fonctionnement des systèmes famille-exploitation (Osty, 1978 ; Landais, 1999 ; Brossier et al., 2003), il apparaît primordial d'identifier l'ensemble des individus qui interviennent sur l'exploitation dans l'étude des transformations des exploitations sur le long terme. Se limiter au "chef d'exploitation » de la terminologie officielle n'est pas suffisant pour comprendre les logiques d'action sur le long terme. La notion de chef d'exploitation a en effet un contour assez flou lorsque l'on s'intéresse à la gestion de l'exploitation agricole familiale (Laurent et Rémy, 2000). D'autres études réalisées dans les Alpes françaises ont montré le rôle de ce collectif familial à travers le collectif de travail (Madelrieux et Dedieu, 2008) ou la lignée (Terrier, 2013). Le jeu des prête-noms en constitue une illustration, mais il nous semble que plus généralement les relations qui se tissent au sein de la famille (entre frères, sœurs, au sein du couple et surtout entre les générations) sont déterminantes pour l'avenir de l'exploitation. La complexité de ces liens n'a toutefois pu être approfondie car, si les entretiens ont eu souvent lieu en présence du couple et d'autres membres de la famille, nous n'avons pas conduit d'entretien individuel avec chaque membre du collectif familial. C'est une démarche qui mériterait d'être mise en œuvre. La compréhension fine du parcours des repreneurs, de leur identité, de leurs motivations pourrait être développée.

Dans tous les cas étudiés ici, le " pilotage stratégique " à long terme des exploitations de la région d'étude semble reposer sur un objectif général ancré dans la société locale (Cantala, 2002 ; Mottet 2005; Gibon et al., 2010) qui vise à assurer la pérennité à long terme de l'exploitation selon des principes et des valeurs proches du modèle culturel de transmission de la " maison ", base traditionnelle d'organisation locale des systèmes famille-exploitation. Les études de cas ont mis en évidence qu'assurer la continuité de la "maison » reste très fréquemment un objectif partagé entre les différents membres de la cellule familiale dans les familles agricoles. Il fait l'objet de nombreuses réflexions et stratégies de la part des différents membres des collectifs familiaux, face aux changements du contexte socio-économique englobant et local (concurrence pour le foncier en particulier).

Si le modèle présenté ici est original par la prise en compte de tout un collectif familial, il présente pour limite de se référer uniquement aux situations de transmission familiale de l'exploitation entre générations. Les transmissions d'exploitation « hors cadre familial " (HCF) observées dans la région (quatre cas sur quarante dans les travaux de Mottet, 2005) ne s'inscrivent pas dans le cadre social de la " maison ", car il s'agit souvent d'agriculteurs venus d'autres régions de France. L'analyse des processus de transmission dans la totalité des exploitations du territoire requiert donc de poursuivre le développement du modèle pour prendre en compte les spécificités de ces HCF. L'avenir des exploitations de la zone dépendra des voies de transmission qui sortent du cercle familial restreint. Le recours à des parents éloignés (Mottet, 
2005 ; Rueff et al., 2012) ou encore à des stagiaires pour trouver un successeur est une alternative à l'absence de reprise par un enfant ou un autre membre de la famille proche.

Pour autant, les travaux de Barthez (1999) sur ces installations HCF ont montré qu'elles ne sortaient pas toujours du cadre d'analyse adapté à des exploitations familiales. En effet, dans certains cas de HCF, des relations de type familial peuvent se mettre en place entre les exploitants et un processus d' " adoption » du jeune installé par les cédants ou les associés peut même avoir lieu. Barthez (1999) a ainsi décrit, comme dans notre modèle, des processus progressifs de cession du capital, de partage de décisions, qui ont lieu surtout dans les HCF ayant mis en place des relations de type familial (plutôt que professionnel) au sein de la société d'exploitation. Les conflits entre générations ou entre exploitants, que nous avons relatés, sont vus dans ses travaux comme des hésitations ou des désaccords quant à l'inscription des relations entre associés dans le monde familial ou professionnel.

\section{Plusieurs dimensions de l'exploitation agricole mises en avant}

Le modèle tridimensionnel présenté dans cette étude met aussi en avant l'intérêt de ne pas regarder uniquement les revenus agricoles (sous l'angle de la viabilité économique), pour comprendre ce qui fait durer une exploitation : la pluriactivité apparaît ici comme un élément déterminant pour expliquer la pérennité des exploitations sur le long terme. La pluriactivité apparaît dans la littérature sous deux angles opposés : elle constitue fréquemment un moyen d'assurer la continuité des exploitations en milieu difficile (De Haan et Zoomers, 2005) ; mais elle représente, dans certains cas, une porte de sortie progressive de l'élevage et de l'agriculture en général. Cette vision ambivalente de la pluriactivité était présente chez les exploitants.

Le modèle élaboré ici ne constitue qu'un point de départ pour l'étude des transformations du tissu local des exploitations dans le territoire étudié, et sa généricité est à tester dans d'autres situations régionales. Une perspective intéressante serait de développer l'analyse de la logique de gestion à long terme du patrimoine agricole " matériel et immatériel ». Par exemple, les travaux de Fiorelli (2007) portant sur le sens du travail agricole chez les agriculteurs pluriactifs, nous semblent rejoindre les analyses et les réflexions faites ici - ainsi que celles de Mottet (2005) et Gibon et al. (2010) - sur la façon, pour les éleveurs pyrénéens, de trouver et choisir un repreneur agricole. S’intéresser aux différentes valeurs attachées par les agriculteurs au travail agricole (et aux activités d'élevage plus spécifiquement) et aux modalités de gestion des agroécosystèmes et du matériel animal, peut permettre d'aller plus loin dans la compréhension de ce fameux "fil d'Ariane " de la pérennité des entreprises (Mignon, 2001). Ce que, dans ses travaux sur des exploitations agricoles, Macombe (2006) a appelé "l'éthique du métier ", et dont elle a montré qu'elle est un des éléments majeurs de la continuité de la gestion à long terme et de la pérennité d'une exploitation agricole. 


\section{Conclusion}

En fait, si l'on revient sur l'essai de compréhension de ce que l'on transmet, quand on transmet une exploitation agricole dans la région étudiée, il apparaît clairement qu'il ne s'agit pas seulement de transmission à l'un de ses enfants d'une entreprise et d'un ensemble de moyens de production qui lui permettent, sans coup férir, de pratiquer et développer une activité professionnelle rentable. Il s'agit aussi de transmettre un patrimoine agricole, foncier, immobilier... mais aussi un mode de vie, des valeurs. Dans le cas du Sud-Ouest, ces transmissions d'exploitations s'inscrivent dans une société rurale locale marquée par le cadre de la " maison ". À chaque génération, le futur gestionnaire est ainsi soigneusement "sélectionné " par le groupe familial (Augustins, 1977). Il apparaît comme un maillon dans une longue chaîne temporelle qu'il faut éviter de rompre. La transmission des savoirs et savoir-faire agropastoraux nécessaires à une mise en valeur et gestion des terres et une conduite des troupeaux permettant de mener à bien ce projet dans la durée est, elle aussi, essentielle dans un tel schéma. Bien qu'il ne nous ait pas été possible de développer la question dans le cadre des limites de ce chapitre, les entretiens ont montré que différentes pratiques sociales destinées à transmettre ces derniers aux enfants, dès le plus jeune âge, sont en vigueur dans certaines familles.

Ce travail contribue aussi, nous semble-t-il, à éclairer la compréhension des logiques d'action des agriculteurs sur le long terme (Dedieu et al., 2008). Il offre une illustration, dans des situations concrètes, de la manière dont les décisions du collectif familial sont mises en œuvre à différents pas de temps. Ceci pour assurer conjointement la pérennité de l'exploitation familiale et des moyens d'existence de la famille grâce à des comportements adaptatifs de divers ordres (stratégie de sécurisation du foncier, saisie d'opportunités offertes par les politiques publiques, pratique de la pluriactivité comme variable d'ajustement dans un projet à long terme...). Ces comportements décisionnels et ces pratiques peuvent être lus comme autant d'illustrations de pratiques de gestion à long terme contribuant à assurer la résilience des exploitations vues comme des systèmes socio-écologiques (Milestad et Darnhofer, 2003 ; Gibon et Hermansen 2006).

\section{Remerciements}

Cette étude a bénéficié d'un financement du projet ANR Trans (2007-2009) du programme ANR «Agriculture et développement durable » et du projet Chapay (2008-2011) du programme PSDR INRA-Région Midi-Pyrénées. Nous remercions chaleureusement les agriculteurs du canton d'Argelès-Gazost (65) qui ont participé aux études de cas. Ce travail doit beaucoup à la qualité de l'accueil qu'ils nous ont réservé. Enfin, nous tenons à exprimer notre gratitude à Catherine Macombe et Sophie Mignon pour leur appui lors de la définition des orientations de l'étude. 


\section{Bibliographie}

Augustins, G., 1977. Reproduction sociale et changement social : l'exemple des Baronnies, Revue française de sociologie, 18, 3, 465-484.

Bailhe, M.-L., 2010. Étude des trajectoires d'évolution des exploitations d'élevage de montagne en référence aux politiques publiques et aux marchés (étude de cas dans les Pyrénées Bigourdanes), Mémoire DAA SPET INP-ENSAT, Toulouse.

Barthez, A., 1999. Installation " hors du cadre familial " et relation d'adoption, Économie rurale, 253, 15-20.

Brossier, J., Chia, E., Marshall, E., Petit, M. (Eds), 2003. Gestion de l'exploitation agricole familiale. Eléments théoriques et méthodologiques, Dijon, éditions Educagri.

Cantala, E. 2002. Élaboration d'un protocole d'étude de la dynamique des pratiques agricoles en référence à la dynamique des boisements spontanés de frênes dans la zone périphérique du parc national de Pyrénées, Mémoire de fin d'études ESAP, Toulouse.

Capillon, A. 1993. Typologie des exploitations agricoles, contribution à l'étude régionale des problèmes techniques, Thèse de doctorat INA P-G, Tomes I et II, Paris.

Cialdella, N. 2005. Stratégies d'élevage dans les projets familiaux en milieu aride. Usages des ressources locales pour gérer l'incertain, cas de la Jeffara (Sud-Est tunisien), Thèse de doctorat de l'INA-PG, Paris.

Cialdella, N., Dobremez, L. Madelrieux, S. 2009. Livestock farming systems in urban mountain regions Differentiated paths to remain in time, Outlook on Agriculture, 38, 2, 127-135.

De Bortoli D., 2007 Le système maison : un déterminant de la pérennité de l'exploitation agricole en Soule, Document de travail WP2, Transformations de l'élevage et dynamiques des espaces, ANR TRANS, Dijon.

Dedieu, B., Faverdin, P., Dourmad, J.-Y., Gibon, A., 2008. Système d'élevage, un concept pour raisonner les transformations de l'élevage, INRA Productions Animales, 21, 1, 45-58.

De Haan, L., Zoomers, A., 2005. Exploring the frontier of livelihoods research, Development and change, 36, 27-47.

Delattre P., 1995. "Interdisciplinaires (recherches) ", Encyclopaedia Universalis, 433-438.

Fiorelli, C., Porcher, J., Dedieu, B. 2007. Pourquoi faire de l'élevage quand on a un autre travail ? in Rencontres autour des Recherches sur les Ruminants, 14, 389392.

Gambino, M., Laisney, C., Vert, J., (Coord.), 2012. Le monde agricole en tendances. Un portrait social prospectif des agriculteurs, Centre d'études et de prospectives, SSP, ministère de l'Agriculture, de l'Alimentation, de la Pêche, de la Ruralité et de l'Aménagement du territoire, La Documentation française.

Gasson, R., Errington, A., 1993. The farm family business, Wallingford, Oxon (UK), CABI Publishing. 
Gibon, A. 2003. Les systèmes d'élevage en ferme. Un champ en émergence de la zootechnie entre filière et territoire, Thèse pour l'obtention de l'habilitation à diriger les recherches (HDR), Institut national polytechnique de Toulouse.

Gibon, A., Di Pietro, F., Theau, J.-P., 1999. La diversité des structures spatiales des exploitations pyrénéennes, Options Méditerranéennes, Série B., 27, 259-266.

Gibon, A., Hermansen, J.-E., 2006. Sustainability concept in LFS research orientations. Conference Proceeding of the EAAP meeting, Antalya, Turkey, Session 1 : Ethics of Sustainability : 1-12.

Gibon, A., Sheeren, D., Monteil, C., Ladet, S., Balent G. 2010. Modelling and simulating change in reforesting mountain landscapes using a social-ecological framework, Landscape Ecology, 25, 2, 267-285.

Jollivet, M. (Eds), 1992. Sciences de la nature, Sciences de la société. Les passeurs de frontières, CNRS éditions, 589 p. URL : http://books.openedition.org/ editionscnrs/4154.

Kaufmann, J.-C. (Eds) 2001. L'entretien compréhensif, Paris, éditions Nathan, collection 128.

Lamaison, P., 1987, La notion de maison : entretien avec C. Lévi-Strauss, Terrain, 9, 34-39.

Landais, E., 1999. Agriculture durable et plurifonctionnalité de l'agriculture, Fourrages, 317-331.

Laurent, C., Rémy, J., 2000. L'exploitation agricole en perspective, Le Courrier de l'environnement de l'INRA, 41.

Legay, J.M., 1996. L'expérience et le modèle : un discours sur la méthode, Versailles, éditions Quae.

Le Moigne J.-L., 1994. La théorie du système général. Théorie de la modélisation, Paris, Presses Universitaires de France.

Macombe, C., 2006. Éthique du métier et transmission des exploitations, La Revue des sciences de gestion, 129-138.

Madelrieux, S., Dedieu, B., 2008. Qualification and assessment of work organisation in livestock farms, Animal, 2, 3, 435-446.

Manoli, C., 2008. La place de la transmission dans les trajectoires des exploitations agricoles. Mise au point d'une méthode d'analyse et application à une vallée des Pyrénées, Rapport de Master Recherche AgroParisTech. Paris.

Mélèse, J., 1972. Analyse modulaire des systèmes, Éd. Hommes et Techniques.

Milestad, R., Darnhofer, I., 2003. Building farm resilience : The prospects and challenges of organic farming, Journal of Sustainable Agriculture, 22, 3, 81-97.

Mignon, S., 2001. Stratégie de pérennité d'entreprise, Paris, éditions Vuibert.

Mottet, A., 2005. Transformations des systèmes d'élevage depuis 1950 et conséquences pour la dynamique des paysages dans les Pyrénées, Thèse pour l'obtention de doctorat, Toulouse, École Doctorale SEVAB.

Mottet, A., Ladet, S., Coqué, N., Gibon A., 2006. Agricultural land-use change and its drivers in mountain landscapes : A case study in the Pyrenees, Agriculture, Ecosystems \& Environment, 114, 2-4, 296-310. 
Moulin, C.H., Ingrand, S., Lasseur, J., Madelrieux, S., Napoléone, M., Pluvinage, J., Thénard V., 2004. Comprendre et analyser les changements d'organisation et de conduite de l'élevage dans un ensemble d'exploitations : propositions méthodologiques, in Dedieu, B., Chia, E., Leclerc, B., Moulin, C.H., Tichit, M. (Eds), Lélevage en mouvement. Flexibilité et adaptation des exploitations d'herbivores, Versailles, éditions Quae, 181-196.

Osty, P.-L., 1978. L'exploitation agricole vue comme un système. Diffusion de l'innovation et contribution au développement, Bulletin Technique d'Information, 326, 43-49.

Perrot, C., 1990. Typologie d'exploitations construites par agrégation autour de pôles définis à dire d'experts. Proposition méthodologique et premiers résultats obtenus en Haute-Marne, Inra Productions Animales, 3, 51-66.

Ponchelet, D., Bellon, S., 1999. Ménages pluriactifs et retraités : une majorité d'agriculteurs dans la vallée de Duyes (Alpes de Haute-Provence), Options Méditerranéennes B, 27, 125-137.

Rueff, C., Choisis, J.-P., Balent, G., Gibon, A., 2012. A preliminary assessment of the local diversity of family farms change trajectories since 1950 in a Pyrenees mountains area, Journal of Sustainable Agriculture, 36, 5, 564-590.

Ryschawy, J., Choisis, N., Choisis, J.-P., Gibon, A., 2013. Paths to last in mixed crop-livestock farming : lessons from an assessment of farm trajectories of change, Animal, 7, 4, 673-681.

Sourdril, A., 2008. Frontières sociales, frontières spatiales. 2008. Approche anthropologique de l'exploitation et de la transmission des petites forêts privées fragmentées des coteaux de Gascogne (Haute-Garonne, Thèse de doctorat de l'Université de Paris X, Nanterre.

Sourdril, A., Ladet, S., 2008. Le paysage d'une "société à maison " bas-commingeoise vu au travers des archives cadastrales et photographiques : quand ethnologie et géomatique s'en mêlent, Ateliers du LESC, URL : http://ateliers. revues.org/3332, mis en ligne le 21 août 2008.

Terrier, M., 2013. Réalités de l'exploitation agricole familiale au prisme du temps long. Proposition d'un cadre d'analyse interdisciplinaire et illustrations en exploitations d'élevage bovin lait dans le Vercors, Thèse de doctorat en Zootechnie des systèmes d'élevage et Sociologie, AgroParisTech, Paris. 
